\title{
CEREBELLO-THALAMO-SPINAL DEGENERATION IN INFANCY : AN UNUSUAL VARIANT OF WERDNIG-HOFFMANN DISEASE
}

\author{
BY \\ R. M. NORMAN and J. M. KAY* \\ From the Burden Neuropathological Laboratory, Frenchay Hospital, Bristol, and the \\ Department of Pathology, University of Bristol
}

(RECEIVED FOR PUBLICATION SEPTEMBER 22, 1964)

The stereotyped clinical picture of hypotonic muscular weakness presented by the majority of children suffering from Werdnig-Hoffmann disease gives little indication of the wealth of neuropathological findings which examination of the brain may sometimes reveal. In such cases the lesions found in the spinal white matter, the cerebellum, or the basal ganglia remain clinically silent because they are masked by the widespread degeneration of the anterior horn cells.

The patient to be described in this paper was an exception to this rule, for spasticity rather than hypotonia was the salient feature, and the diagnosis of Werdnig-Hoffmann disease was not suggested during life. Yet neuropathological changes characteristic of this disease were present, and the atypical clinical picture must be attributed to the unusual distribution of the lesions.

\section{Case Report}

K.M., a girl, was the third child of healthy parents who were not blood relatives.

The first child, a boy, came under observation at the age of 11 months because of his failure to sit up or take interest in his surroundings. Examination showed a convergent strabismus, a generalized increase in muscle tone, which was more pronounced in the lower limbs, brisk reflexes, and a head circumference of 18.75 in. $(48 \mathrm{~cm}$.). Pneumoencephalography was performed when the child was 18 months: this showed a mild degree of symmetrical hydrocephalus probably compensatory to cerebral hypoplasia or atrophy. He was admitted to a home for mentally retarded children where he died at the age of 28 months. At necropsy the head circumference was 19 in. $(48 \mathrm{~cm}$.), the cerebral white matter was thin, the ventricles were dilated, and the subarachnoid space increased in width. No microscopical examination was made.

The second child is a normal healthy girl.

* Present address: Department of Pathology, University of Birmingham.
K.M. was born after an uneventful full-term pregnancy and a normal delivery. She was admitted to hospital for investigation at the age of 11 months because of failure to reach her milestones, difficulty in feeding, and failure to gain weight. On examination it was found that she was unable to sit up and could not roll from the prone to the supine position. The head circumference measured $17 \cdot 25$ in. $(44 \mathrm{~cm}$.), the weight was $16 \cdot 75 \mathrm{lb} .(7 \cdot 6 \mathrm{~kg}$.) and length 27 in. $(69 \mathrm{~cm}$.). Muscle tone was generally increased but more so in the lower limbs. The thumbs were maintained abducted across the palms. The cranial nerves were normal. The tendon reflexes were uniformly brisk. An extensor plantar response was elicited on the left side. Examination of the urine for phenylketones, amino acids, reducing substances, and metachromatic fat granules yielded negative results. Serological tests for syphilis and toxoplasmosis were negative. Radiological examination of the skull showed no abnormality. An electroencephalogram taken on this admission was normal. The child's condition remained virtually the same until the age of 22 months when she was admitted for further investigation. At this time her head circumference was 18 in. $(46 \mathrm{~cm}$.), her weight was $16 \mathrm{lb}$. $(7 \cdot 3 \mathrm{~kg}$.) and length 27 in. $(69 \mathrm{~cm}$.). She could not sit up, but lay in bed with her legs semiflexed at the knee and hip joints. An electroencephalogram recorded extremely low potential, slow components from the right hemisphere and virtually nothing from the left side of the head, which was interpreted as indicating an atrophic or dystrophic condition of the brain. Shortly after admission she developed a high temperature for which no cause could be found; penicillin was administered and the temperature subsided. Twelve days later, however, she suddenly collapsed and died.

Necropsy. A full necropsy revealed no abnormalities outside the nervous system. Microscopically the lungs, heart, spleen, liver, pancreas, and psoas major muscle showed no significant pathological changes. A cause for the child's sudden death could not be found.

\section{Neuropathology}

MACroscopic. The fixed brain weighed $880 \mathrm{~g}$., of 


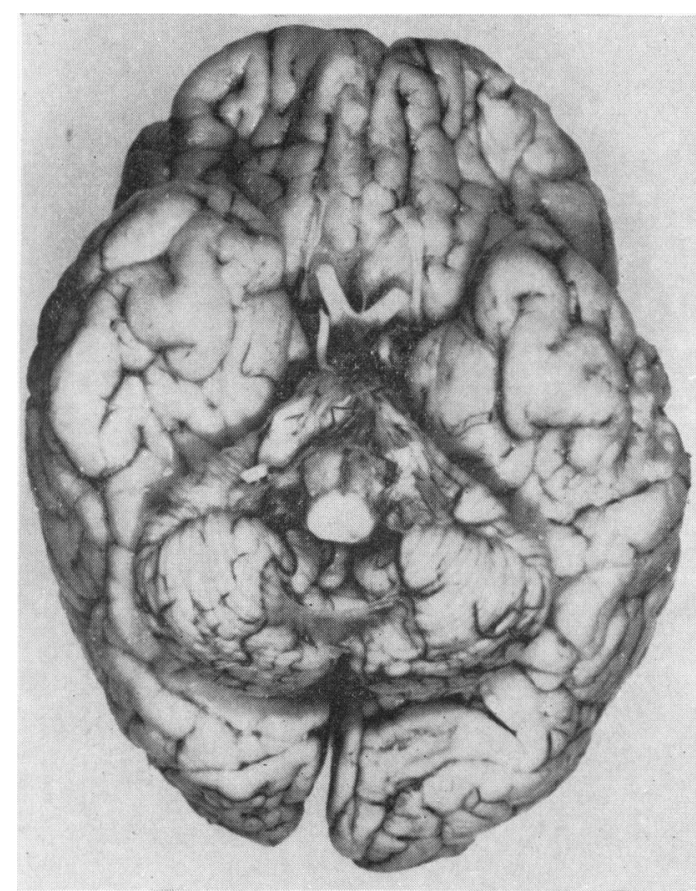

Fig. 1.-Base of brain showing small size of cerebellum and widely separated lateral lobes.

which the cerebellum and brain-stem accounted for $49 \mathrm{~g}$. (normal 120 g.). The convolutional pattern, cranial nerves, and basal vessels were normal. On coronal

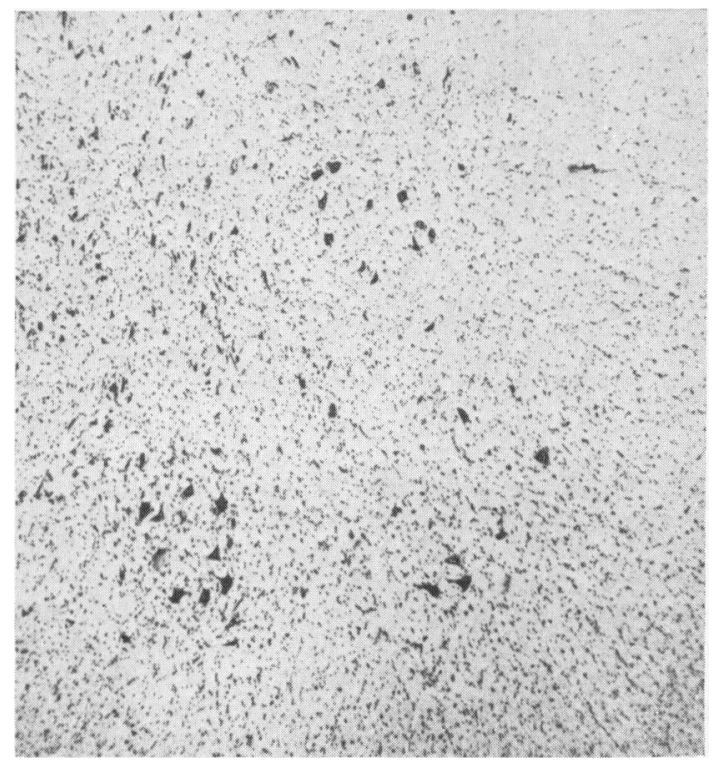

FIG. 2.-C7. Anterior horn showing poverty of nerve cells in the antero-lateral area. The antero-median cell group is well preserved. (Carbol azure. $\times$ 45.) section the ventricles were slightly enlarged and the thalami appeared small. There was no hypoplasia of the corpus callosum. The cerebellum was small, and there was an undue separation of the lateral lobes which allowed the tonsils and inferior vermis to be easily seen (Fig. 1).

Microscopic. Representative parts of the cerebral hemispheres, cerebellum, brain-stem, and spinal cord were stained by standard methods in celloidin and frozen sections. The cerebellum, brain-stem, and segments of the cervical and lumbar cord were studied in spaced serial sections.

Spinal Cord. In the cervical region the anterior horn cells were reduced in number, the amount of neuronal loss varying considerably in the individual cell groups at different levels (Fig. 2). In most sections there were one to five degenerating nerve cells of ovoid or round shape which had lost their Nissl bodies and stained a pale, uniform blue, the nucleus retaining its neucleolus and membrane, and being usually displaced to the margin of the cell (Fig. 3a). Sometimes a remnant of the Nissl substance was present at the periphery of the cyton (Fig. 3b). Microglial nuclei were often abnormally numerous but there were no figures of neuronophagia. In the thoracic cord the loss of nerve cells was less conspicuous. Clarke's column was preserved in the thoracic segments which were sampled, one chromatolytic cell being seen. At the level of L1, however, there was almost complete loss of nerve cells and several greatly inflated chromatolytic cells (Fig. 3c). The myelin picture showed the normal configuration of the nucleus with spaces devoid of nerve cells ('empty cell beds'). The lumbar enlargement seemed at first sight normal in its neuronal population, but here and there, at different levels, the same distinctive chromatolytic change was found in isolated cells (Fig. 3d).

In sections stained for myelin the cervical cord showed a marked pallor in the lateral columns and a slight pallor at some levels in the columns of Goll (Fig. 4a). In the thoracic segments the myelin stained poorly in the whole cord except in the posterior columns, and in the lumbar region the pallor was confined to the pyramidal tracts. Fibrous gliosis was increased throughout the cord, the ventral part of the anterior horns being specially affected (Fig. 4b). In the white matter the gliosis was usually but not invariably accentuated in the areas of myelin pallor. In the cervical region the columns of Goll were distinguished by the denser gliosis present in them. There were no sudanophil products of myelin breakdown, and the axis cylinders in transverse and longitudinal sections showed no significant differences from those in a normally myelinated cord coming from a 2-year-old epileptic child. The nerve roots appeared normal except in the cervical region where the anterior roots were often extremely small and contained less densely packed myelinated fibres than the posterior roots (Fig. 4a).

Brain-stem. Both superior vestibular nuclei showed a loss of nerve cells and contained several swollen, chromatolytic cells analogous to those in the spinal cord (Fig. 5a). The hypoglossal nucleus on one side also showed a patch of neuronal loss with one degenerating nerve cell. There 


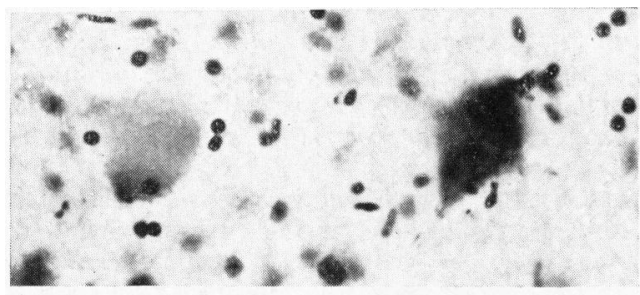

(a)

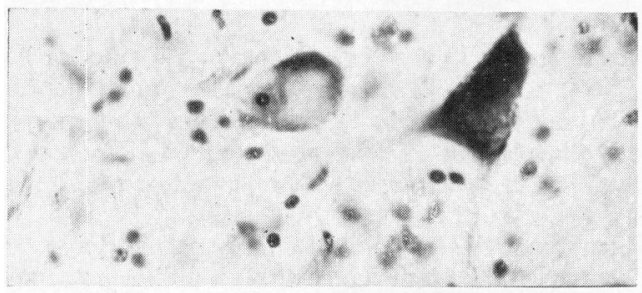

(b)

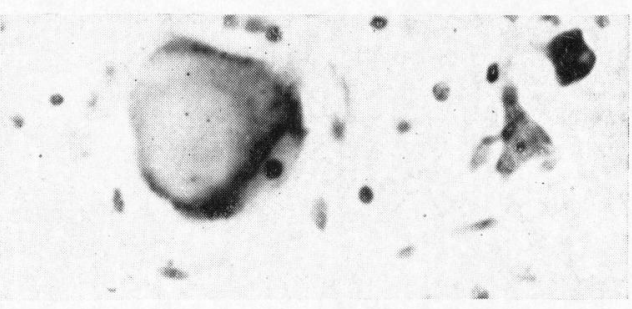

(c)

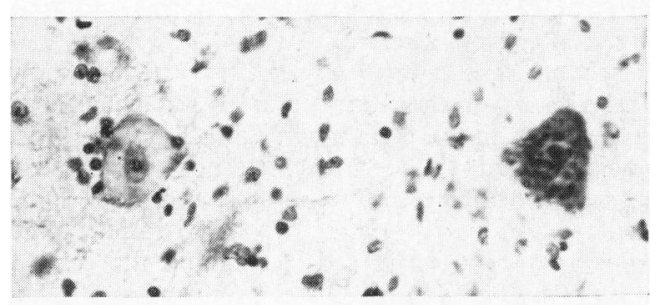

(d)

Fig. 3-Chromatolytic anterior horn cells. (a) and (b) from the cervical region; (c) from Clarke's column at L1 level (all $\times 400)$ and (d) from the lumbar region $(\times 282$.)

was a marked increase in fibrous neuroglia throughout the brain-stem, both in the nuclei and fibre tracts, but the myelination was unaffected. The medullary pyramids and pes pontis were of normal size.

Basal Ganglia. Severe pathological changes were found in the thalami where large parts of the lateral nuclei were almost devoid of nerve cells and showed marked fibrous gliosis (Fig. 6). The fibres of passage were normally myelinated. The principal anterior nuclei also showed widespread degeneration while the accessory anterior nucleus of each side had retained most of its cells

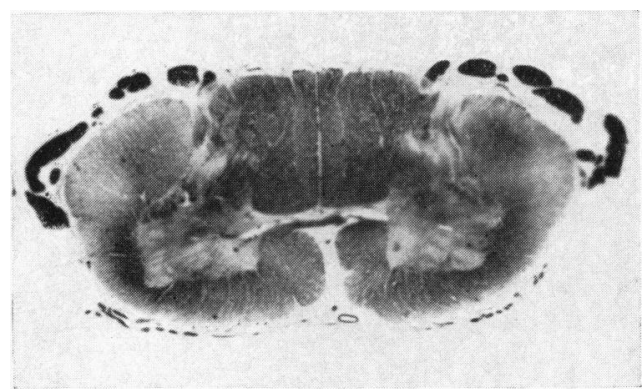

(a)

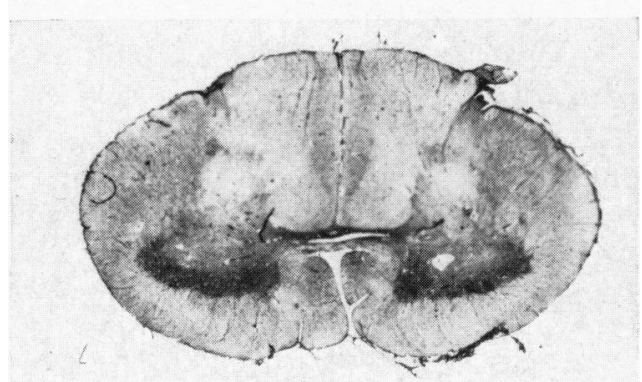

(b)

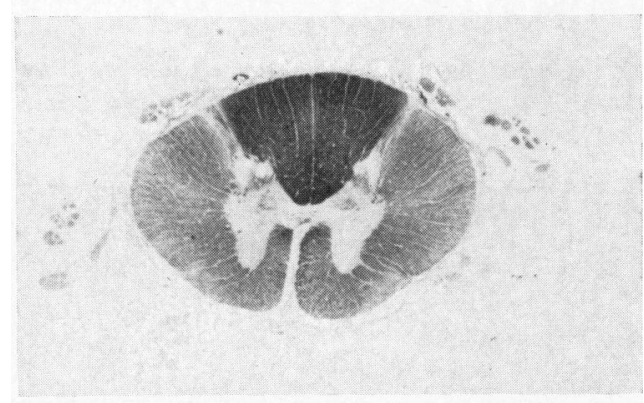

(c)

FIG. 4.-(a) Cervical cord showing pallor predominantly in the lateral columns, and atrophy of the anterior roots. (Heidenhain. $\times 5 \cdot 4$.) (b) Cervical cord showing gliosis in the anterior horns, lateral columns and tracts of Goll. (Holzer. $\times 4 \cdot 8$.) (c) Thoracic cord showing normal myelination only in the posterior columns. (Heidenhain. $\times 7 \cdot 3$.

(Fig. 7). Patchy nerve cell loss and gliosis were present in the medial nuclei and in the pulvinars. In the partially degenerated areas there were several slightly swollen neurones that had completely lost their Nissl substances and stained a uniform pale blue (Fig. 5b). These chromatolytic cells were sharply distinguishable from the healthy nerve cells which greatly outnumbered them There was little microglial activity except for a few clusters suggestive of neuronophagia. One binucleate nerve cell was seen. A few chromatolytic cells were found in the external geniculate body. The corpus striatum, subthalamic nucleus, and globus pallidus of 


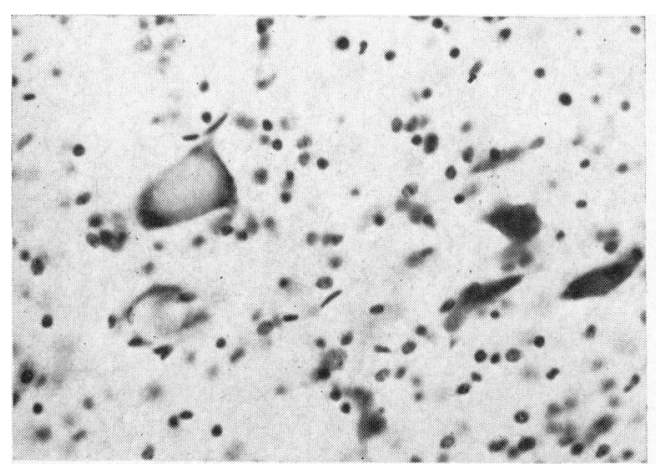

(a)

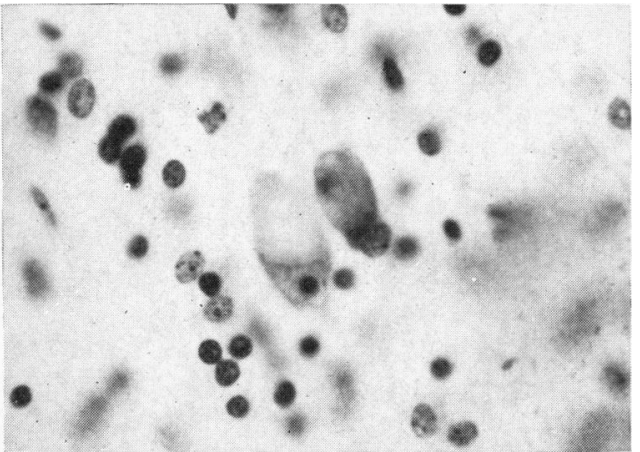

(b)

Fig. 5.-(a) Chromatolytic nerve cell in the superior vestibular nucleus. (Carbol azure. $\quad \times 263$.) (b) Chromatolytic cell in the pulvinar of the thalamus. (Carbol azure. $\times 518$.)

each side were normal except for a mild fibrous gliosis in the latter nucleus, which was unaccompanied by myelin loss.

Cerebellum. The granular layer of the cortex of the lateral lobes and vermis showed a diffuse reduction in cellular density. Very few healthy Purkinje cells were seen, the great majority presenting the appearance of simple atrophy with marked shrinkage of the nucleus and cell body, the latter often showing vacuolation (Fig. 8a). There were only minor losses of Purkinje cells, and usually the apparent gaps in this layer contained barely visible remnants of atrophic neurones. Silver impregnation showed that the dendrites of the remaining cells and the tangential and basket fibres were present, but the

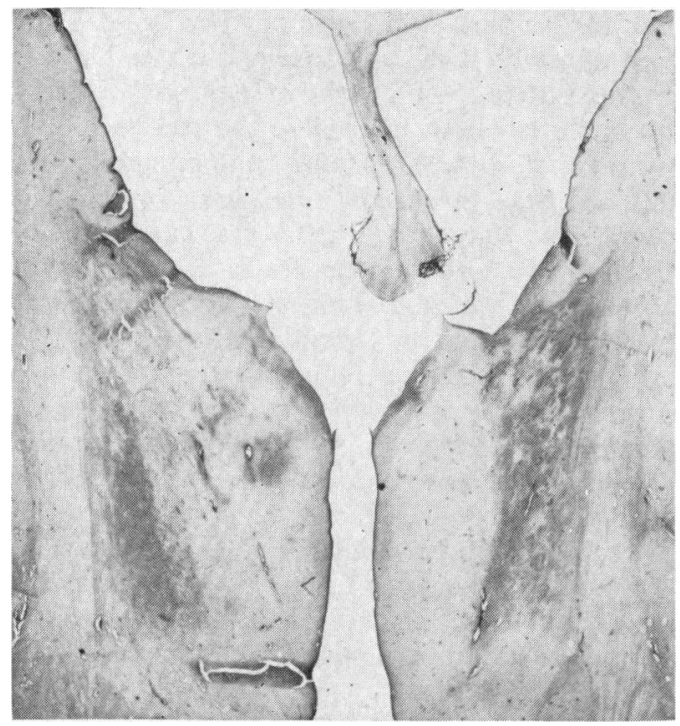

Fig. 6.-Thalami showing dense fibrous gliosis in the lateral nuclei. (Holzer. $\times 3.7$.) latter were often feebly represented. There was a marked fibrous gliosis in the whole of the central white matter and the folial cores, and a moderate proliferation of the Bergmann fibrils throughout the molecular layer. A slight pallor of myelin staining was seen in the posterior part of the left lateral lobe and also in the hila of both dentate nuclei where gliosis was particularly intense (Fig. 9). The nerve cells of the dentate nuclei showed considerable shrinkage but no numerical reduction. Several chromatolytic cells and a few figures of neuronophagia were seen in the nucleus emboliformis.

Other Parts of the Brain. The cerebral cortex, including the hippocampal formations, were normal in every respect. A slight fibrous gliosis without detectable

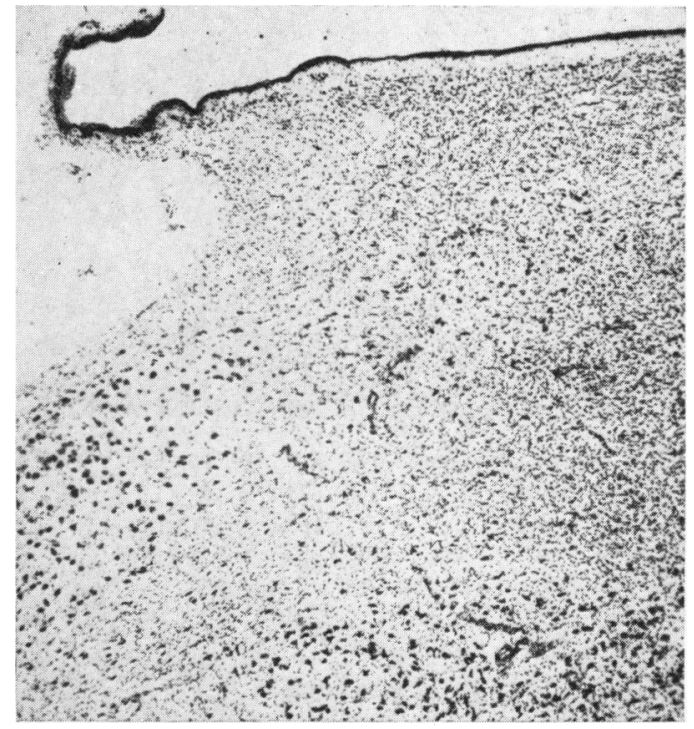

FIG. 7.-Thalamus showing almost complete loss of nerve cells and greatly increased glial nuclei in the principal anterior nucleus. Cells are seen in the accessory anterior nucleus (left). (Carbol azure. $\times 3 \cdot 3$.) 


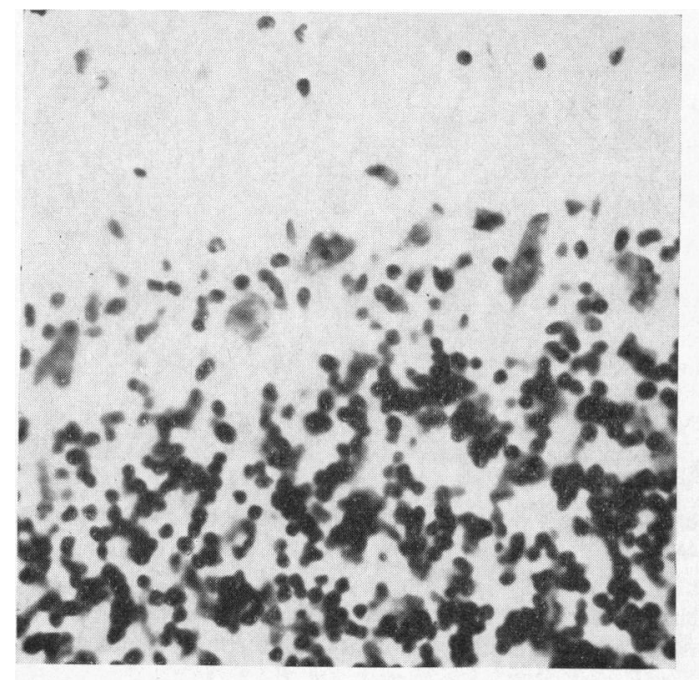

(a)

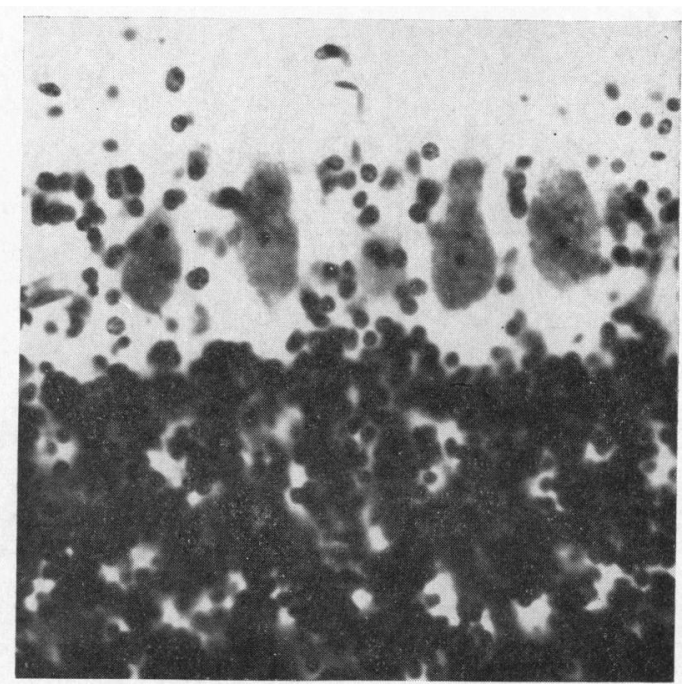

(b)

FIG. 8.-(a) Cerebellar cortex showing rarefied granular layer and atrophy of Purkinje cells (b) Normal cerebellar cortex in child of 2 years. (Carbol azure. $\times 382$.)

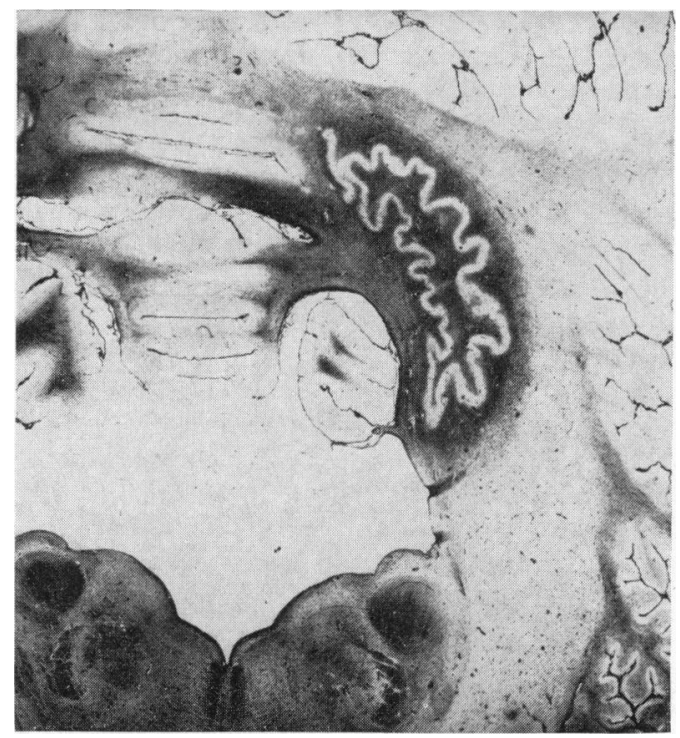

FIG. 9.-Fibrous gliosis in the dentate nucleus and brain-stem. (Holzer. $\times 3 \cdot 5$.)

defect of myelin was present throughout the centrum ovale, gyral cores, optic nerves, and tracts. The choroid plexuses, ependyma, leptomeninges, and blood vessels were normal.

\section{Discussion}

The diagnosis of Werdnig-Hoffmann disease in this case rests on the neuropathological findings, since persistent spasticity of the legs and a positive
Babinski response have never been described in this condition. Nevertheless, the classical features of the disease were seen in the loss of anterior horn cells, the atrophy of the anterior roots, and the presence of swollen, chromatolytic nerve cells, not only in the spinal cord, but in some of the brain-stem nuclei and the thalamus. It has been realized since the time of Conel's papers (Conel, 1938, 1940) that the latter change is more characteristic of the disease than small size or dark staining of nerve cells, the significance of which is difficult to assess in the immature nervous system. This form of neuronal degeneration has often been likened to the central chromatolysis that occurs after axonal lesions and it has been aptly named gonflement bulleuse by Thieffry, Arthuis, and Bargeton (1955). One cannot say that this form of neuronal degeneration per se is pathognomonic of Werdnig-Hoffmann disease, but when the distribution of the lesions is taken into account, we know of no other heredo-degeneration occurring in this age-group with which the condition might be confused. Except in Werdnig-Hoffmann disease we have only once seen acute anterior horn cell changes of approximately the same type, namely, in a child of 2 years with precocious Friedreich's ataxia (as described by André-van Leeuwen, 1949), and in this case there were the distinguishing features of degeneration of the posterior columns and of the visual pathways.

An atypical feature of the present case, and one that seems to be partly responsible for the clinical condition, was the predominant involvement of the 
cervical in contrast with the lumbar region of the cord. There is some evidence that this disease may start in certain segments and even become localized in them for a considerable time. Thus De Lange (1937) found intact lumbar segments in an infant who died on the 20th day of life, and Thieffry et al. (1955) noted that muscular weakness was confined to the lower limbs in 2 of the 40 patients they studied. The relative integrity of the lumbar cord in our patient, combined with the lesions present in the lateral columns, may be held responsible for the spasticity observed during life. These changes in the white matter, though unusually extensive, were similar in type to those often described in other cases of Werdnig-Hoffmann disease, i.e. pallor of myelin staining, not sharply confined to individual tracts and varying somewhat in intensity, in distribution, and in the associated gliosis, according to the level examined. Radermecker (1951), in particular, has drawn attention to these lesions which he believes are degenerative in character, but in view of the changes described in our case it seems more likely that defective myelination had occurred.

The exceptionally severe atrophy of the thalami and cerebellum in this brain invites comparison with the suprasegmental lesions found in the case recently reported in this journal by Norman (1961). In this instance, the cerebellum weighed only $21 \mathrm{~g}$. and showed a combination of malformation (retarded convolutional development) and atrophy, which was regarded as an early manifestation of Werdnig-Hoffmann disease. This interpretation did not go unchallenged, for after the publication of the paper more than one correspondent suggested that birth injury was a more plausible explanation for the lesions, particularly in view of the clinical history. This opinion failed to take into account the acute neuronal changes in the spinal cord and basal ganglia which were typical of WerdnigHoffmann disease, but at that time the possibility of a double pathology could not be entirely ruled out. The present case removes any lingering doubt as to the propriety of attributing such severe suprasegmental lesions to a degenerative disease. There was no clinical suggestion of birth trauma, and the cerebellar cortical changes were those of a diffuse degenerative process of slow tempo which had introduced an element of hypoplasia into the postnatal growth of the organ. The exceptionally severe loss of nerve cells in the thalami may have accounted for the abnormalities shown in the second electroencephalogram.

In conclusion it may be pointed out that the brother of this patient had died at about the same age and had also shown spasticity and mental retarda- tion. At necropsy the dilated lateral ventricles with no associated enlargement of the head had suggested an atrophic process, but since the brain was not examined histologically no further comparison is possible. However, it seems likely that both children suffered from the same genetic condition. The final diagnosis depends upon the propriety of including such atypical cases within the category of Werdnig-Hoffmann disease. For reasons already given, we believe that our patient did, in fact, suffer from an unusual variant of this disease. The neuropathological findings add no new dimension to its definition: they merely confirm the wide range of variation that may exist in the distribution and severity of the degenerative process.

\section{Summary}

A girl aged 23 months at death had failed to achieve normal motor development and was regarded clinically as a case of spastic cerebral palsy of unknown origin. Another sib had died at 28 months with similar clinical features. Neuropathological examination in the first case showed acute changes in the anterior horn cells, brain-stem nuclei, and thalamus, which were histologically typical of Werdnig-Hoffmann disease. The spinal cord changes were atypical in so far as the cervical segments were much more affected than the lumbar as regards loss of anterior horn cells. In myelin preparations, the spinal cord showed marked pallor of the antero-lateral areas, which was interpreted as the consequence of defective myelination rather than true tract degenerations. Supra-segmental lesions were particularly impressive in this brain. The cerebellum was small and showed mild diffuse atrophy of the granular layer, marked shrinkage of the Purkinje cells, gliosis of all the cortical layers and of the central white matter, especially in the hila of the dentate nuclei. The thalami showed an extensive loss of nerve cells, with corresponding gliosis, particularly in the lateral nuclei.

It is suggested that this thalamic lesion was responsible for the abnormal EEG recorded in the later stages of this child's illness.

These findings have been compared with those in a previously reported case of Werdnig-Hoffmann disease, which also showed unusually severe suprasegmental lesions.

We wish to thank Professor A. V. Neale and Professor T. F. Hewer for their kindness in making their records available to us.

\section{REFERENCES}

André-van Leeuwen, M. (1949). Sur deux cas familiaux de maladie de Friedreich avec atrophie optique précoce globale et grave (observation de dix années). Rev. neurol., 81, 941. 
Conel, J. L. (1938). Distribution of affected nerve cells in a case of amyotonia congenita Arch. Neurol. Psychiat. (Chic.), 40, 337.

(1940). Distribution of affected nerve cells in amyotonia congenita (second case). Arch. Path., 30, 153.

De Lange, Cornelia (1937). Studien über angeborene Lahmungen bzw. angeborene Hypotonie. Acta paediat. (Uppsala), 20, Suppl. 3.
Norman, R. M. (1961). Cerebellar hypoplasia in Werdnig-Hoffmann disease. Arch. Dis. Childh., 36, 96.

Radermecker, J. (1951). L'amyotrophie spinale de l'enfance (Werdnig-Hoffmann) comme hérédo-dégénérescence. Rev. neurol., 84, 14.

Thieffry, S., Arthuis, M., and Bargeton, E. (1955). Quarante cas de maladie Werdnig-Hoffmann avec onze examens anatomiques. ibid., 93, 621. 